Check for updates

Cite this: Soft Matter, 2019, 15,2178

Received 29th December 2018, Accepted 1st February 2019

DOI: $10.1039 / \mathrm{c} 8 \mathrm{sm} 02634 \mathrm{c}$

rsc.li/soft-matter-journal

\title{
Interfacial properties of lipid sponge-like nanoparticles and the role of stabilizer on particle structure and surface interactions $\dagger$
}

\author{
Maria Valldeperas, (D) ${ }^{\text {ab }}$ Aleksandra P. Dabkowska, (D) C Gunnar K. Pálsson, ${ }^{\text {d }}$ \\ Sarah Rogers, (D) Najet Mahmoudi, (D) ${ }^{e}$ Anna Carnerup, ${ }^{a} J^{e}$ ustas Barauskas ${ }^{f}$ and \\ Tommy Nylander (ID *ab
}

\begin{abstract}
The advantage of using nonlamellar lipid liquid crystalline phases has been demonstrated in many applications, such as drug delivery, protein encapsulation and crystallisation. We have recently reported that a mixture of mono- and diglycerides is able to form sponge-like nanoparticles ( $\mathrm{L}_{3}-\mathrm{NPs}$ ) with large enough aqueous pores to encapsulate macromolecules such as proteins. Here we use small angle neutron scattering (SANS) to reveal morphology, structural and chemical composition of these polysorbate 80 (P80) stabilized sponge phase nanoparticles, not previously known. Our results suggest that $\mathrm{L}_{3}$-NPs have a core-shell sphere structure, with a shell rich in P80. It was also found that even if P80 is mostly located on the surface, it also contributes to the formation of the inner sponge phase structure. An important aspect for the application and colloidal stability of these particles is their interfacial properties. Therefore, the interfacial behaviour of the nanoparticles on hydrophilic silica was revealed by Quartz crystal microbalance with dissipation (QCM-D) and neutron reflectivity (NR). Adsorption experiments reveal the formation of a thin lipid layer, with the dimension corresponding to a lipid bilayer after $L_{3}-N P s$ are in contact with hydrophilic silica. This suggests that the diglycerol monoleate/Capmul GMO-50/P80 particles reorganize themselves on this surface, probably due to interactions between $\mathrm{P} 80$ head group and $\mathrm{SiO}_{2}$.
\end{abstract}

\section{Introduction}

Nonlamellar lipid liquid crystalline nanoparticles (LCNP) are widely considered in many applications due to their welldefined internal structure, stability and easily tuneable properties. Varying the lipid composition allows the formation of stable colloidal particle dispersions with inverse cubic, sponge-phase or hexagonal structure (cubosomes, spongosomes and hexosomes, respectively). ${ }^{1-5}$ In addition, they are advantageous

\footnotetext{
${ }^{a}$ Physical Chemistry, Department Chemistry, Lund University, Lund, Sweden. E-mail: tommy.nylander@fkem1.lu.se

${ }^{b}$ NanoLund, Lund University, Lund, Sweden

${ }^{c}$ Pharmaceutical Sciences, IMED Biotech Unit, AstraZeneca, Gothenburg, Sweden

${ }^{d}$ Institut Laue Langevin, Grenoble, France and Department of Physics, Uppsala University, Uppsala, Sweden

${ }^{e}$ ISIS, STFC, Rutherford Appleton Laboratory, Didcot, UK

${ }^{f}$ Camurus AB, Ideon Science Park, Lund, Sweden

$\dagger$ Electronic supplementary information (ESI) available: Core-shell sphere model; estimated solvent volume fraction and SLD of the lipid/P80 of the core and shell and equations used for the calculation; Voigt viscoelastic model; shear and viscosity values obtained using the Voigt model; QCM-D results at different conditions; and neutron reflectometry fits for the 5-layer model. See DOI: 10.1039/ c8sm02634c
}

compared to micelles or their lamellar analogues (vesicles), as their surface area per volume ratio is larger and they have a larger capability to solubilize both hydrophilic and hydrophobic molecules. $^{3,6,7}$ The wide range of applications of these highly versatile particles includes drug delivery, protein encapsulation or crystallisation. , $^{3-12}$

Most of the LCNP investigated so far had inverse cubic or hexagonal inner structures. More recently, the lipid sponge phase $\left(\mathrm{L}_{3}\right)$ has attracted more attention due to its flexibility and capacity for forming large aqueous pores able to encapsulate large bioactive molecules. ${ }^{5,13,14}$ This is more challenging to obtain with other reverse mesophases (cubic or hexagonal), where the cell dimensions are usually smaller or require the use of organic solvents or specific environmental conditions (temperature or pressure). ${ }^{15-17}$

The interfacial properties of cubosomes and hexosomes have been examined to understand the nature of the interactions between these LCNP and different interfaces that mimic storage or administration vessels, such as glass vials, catheters or syringes. ${ }^{18-22}$ The adsorption behaviour was observed to depend on the environmental conditions ( $\mathrm{pH}$, ionic strength), the surface properties (such as hydrophilicity/phobicity or charge) 
and the lipid system used to form the LCNP. Vandoolaeghe et $a .^{19,23}$ studied the adsorption of glycerol monolein (GMO)/ pluronic F127 cubosomes on hydrophilic and hydrophobic silica at different conditions. They found that intact cubosomes were adsorbed on hydrophilic silica in the presence of electrolytes. In contrast, a lipid monolayer structure was formed on hydrophobic silica independently of the salt concentration. Soy phosphatidylcholine (SPC)/glycerol dioletate (GDO)/polysorbate 80 (P80) LCNP were found to form lipid multilayers on anionic silica that increase in thickness with P80 concentration. ${ }^{22,24}$ However on cationic silica, intact LCNP were found to deposit without being dependent on the P80 concentration.

In order to obtain colloidally stable LCNP with a narrow size distribution, a stabilizer that reduces the LCNP aqueous phase interfacial tension, like Pluronic F127 and P80 is required. It has been proposed earlier that P80 not only acts as a dispersing agent found on the outside of the particles, but also contributes to the inner structure organization of the nonlamellar LCNP. This effect is rather pronounced at high P80 concentrations, where even the loss of internal particle structure can occur. ${ }^{22,25}$ In our earlier work ${ }^{13}$ with the same DGMO/GMO-50/P80/water system as studied here, we were also able to form nanoparticles with highly swollen sponge phase structures over a wider range than in the pure lipid-aqueous system. Cryo-TEM imaging showed that such phases could be dispersed into sponge-like nanoparticles ( $\mathrm{L}_{3}$-NPs) that featured a dense sponge phase core with swollen bilayer structures at the interface. We suggested that P80 was helping in the formation of these structures at the nanoparticle surface and thus, stabilizing the $\mathrm{L}_{3}$-NPs. However, the internal structural and the compositional organisation are still not known. We have here therefore used small angle neutron scattering (SANS) and partial deuteration of the stabilizer to reveal these important properties of the particles. This allowed us to reveal the location of deuterated P80 (dP80), as well as better understand the morphology of these particles as the $\mathrm{L}_{3}$ phase is not as well studied as other lipid liquid crystal (LLC) phases. Furthermore, for the first time interfacial behaviour of $\mathrm{L}_{3}$-NPs on hydrophilic silica were studied by Quartz crystal microbalance with dissipation (QCM-D) and neutron reflectometry (NR). The results suggest that the $\mathrm{L}_{3}$-NPs, that were initially $180 \mathrm{~nm}$ in diameter, spread and rearrange into a thin lipid layer onto hydrophilic silica.

\section{Experimental}

\subsection{Materials}

A mixture of acylglycerides denoted as Capmul ${ }^{\circledR}$ GMO-50 (Lot No. 100616-8) was provided by Abitec (Janesville, WI). Its composition was $54.7 \%$ monoglycerides, $15-35 \%$ diglycerides and $2-10 \%$ triglycerides with the following fatty acid composition: $84.6 \%$ oleic (C18:1), 6.8\% linoleic, $0.8 \%$ linolenic and $6.2 \%$ saturated acids. Diglycerol monooleate (DGMO) with $88 \%$ of diglycerol monoester and $4.9 \%$ free glycerol and polyglycerols was received from Danisco A/S (Brabrand, Denmark). The main fatty acid component was oleic acid constituting $90.7 \%$, followed by linoleic $(4.2 \%)$, saturated $(2.9 \%)$, eicosenoic $(1.2 \%)$ and linolenic $(0.8 \%)$. Polyoxyethylene (20) sorbitan monooleate (polysorbate 80, P80) was supplied by Croda (Chocques, France) for the neutron reflectometry measurements. P80 with hydrogenated and deuterated polyoxyethylene head group (hP80 and dP80), but with hydrogenated oleate chain, used in SANS experiments were synthesised by Oxford Deuteration Laboratory (Dr Robert K. Thomas) as described by Tucker et $a .^{26}$ Milli-Q purified water $(18 \mathrm{M} \Omega \mathrm{cm})$ was used for all experiments and heavy water (SigmaAldrich) was employed for neutron reflectometry and scattering measurements. All other solvent and reagents were of analytical grade and were used as received.

\subsection{Sample preparation}

A stock solution composed of DGMO, GMO-50 and P80 was first prepared by co-melting GMO-50 and P80 at $40{ }^{\circ} \mathrm{C}$ followed by further weighting of each component into a glass vial. The DGMO/GMO-50 ratio was kept at 40/60 while the lipid/P80 ratio was 70/30. The stock solution was left mixing for $24 \mathrm{~h}$ in a roller mixer. Then, sponge-like nanoparticle dispersions were prepared by adding the stock solution into water or heavy water at a concentration of $5 \mathrm{wt} \%$ (lipids + P80). The samples were immediately sealed, vigorously hand-shaken and left for $24 \mathrm{~h}$ on an orbital shaking table at $300 \mathrm{rpm}$ and room temperature. Samples for QCM-D and neutron reflectometry were heated up at $121{ }^{\circ} \mathrm{C}$ and 1.4 bars for $36 \mathrm{~min}$ (Prestige Medical Classic 2100 Autoclave, TG-Instrument AB, Helsingborg, Sweden) to remove vesicular aggregates. Samples for SANS measurement were mixed and diluted in appropriate amounts to obtain different contrast and concentrations as specified below. In order to determine the effect of the solvent medium, i.e. $\mathrm{D}_{2} \mathrm{O}$ versus $\mathrm{H}_{2} \mathrm{O}$, on the particle we prepared a sample in the $1: 4 \mathrm{H}_{2} \mathrm{O}: \mathrm{D}_{2} \mathrm{O}$ contrast using two different protocols. These two equivalent samples of $1 \%$ dilution with identical lipid and P80 composition were prepared by (a) mixing $\mathrm{L}_{3}$-NPs initially prepared in $\mathrm{H}_{2} \mathrm{O}$ with $\mathrm{D}_{2} \mathrm{O}\left(1 / 4 \mathrm{NPs} / \mathrm{D}_{2} \mathrm{O}\right.$ volume) or (b) mixing $\mathrm{L}_{3}$-NPs initially in $\mathrm{D}_{2} \mathrm{O}$ with $\mathrm{D}_{2} \mathrm{O}+\mathrm{H}_{2} \mathrm{O}$ $\left(1 / 3 / 1 \mathrm{NPs} / \mathrm{D}_{2} \mathrm{O} / \mathrm{H}_{2} \mathrm{O}\right.$ volume).

\subsection{Small angle neutron scattering (SANS)}

2.3.1. Measurements. SANS experiments were performed to characterise the structure and form of sponge-like nanoparticles $\left(\mathrm{L}_{3}-\mathrm{NPs}\right)$ and locate the P80 in these nanostructures. For this purpose, deuterated and non-deuterated P80 were used (dP80 and hP80, respectively), as well as deuterated and hydrogenated water $\left(\mathrm{D}_{2} \mathrm{O}\right.$ and $\mathrm{H}_{2} \mathrm{O}$, respectively) and $1: 4 \mathrm{H}_{2} \mathrm{O}: \mathrm{D}_{2} \mathrm{O}$ as bulk solvent contrast. The reason for the $1: 4 \mathrm{H}_{2} \mathrm{O} / \mathrm{D}_{2} \mathrm{O}$ contrast is that it matches the SLD of enzymes, which will be used in future studies. First, 0.5\% dP80 micelles were analysed to reveal the degree of deuteration of P80 head group, followed by the evaluation of $5 \mathrm{wt} \%$ and $1 \mathrm{wt} \% \mathrm{~L}_{3}$-NPs in different contrast. Samples were filtered through a $0.45 \mu \mathrm{m}$ syringe filter (PALL $^{\circledR}$ Acrodisc $^{\circledR} 32 \mathrm{~mm} 0.45 \mu \mathrm{m}$ Supor ${ }^{\circledR}$ membrane) and measured in $1 \mathrm{~mm}\left(\mathrm{H}_{2} \mathrm{O}\right.$ and $\left.1: 4 \mathrm{H}_{2} \mathrm{O}: \mathrm{D}_{2} \mathrm{O}\right)$ or $2 \mathrm{~mm}\left(\mathrm{D}_{2} \mathrm{O}\right)$ path length quartz cuvettes (Optiglass, UK). The experiment was carried out on the time-of-flight SANS instrument, SANS2D, at the ISIS Neutron and Muon source of the STFC Rutherford 
Appleton Laboratory. The measurements were performed at two sample-to-detector distances of $4 \mathrm{~m}$ and $12 \mathrm{~m}$. The wavelength range was $\lambda=2-14 \AA$, which means a total covered $q$-range of $0.00168-0.98 \AA^{-1}$, where $q=4 \pi / \lambda \sin (\theta / 2)$ and $\theta$ is the scattering angle. The data reduction and solvent subtraction was performed as described by Wadsäter et $a l^{22}$

2.3.2. Data evaluation. The data was evaluated using the SASView software. ${ }^{29}$ The DREAM algorithm was employed for the fitting of the models to ensure that the equilibrium distribution was reached for the calculations. This algorithm provided the quantification of the uncertainty, which reflects the goodness of the fit. P80 micelles were fitted by using a core-shell sphere form factor, where interparticle correlations were considered negligible $(S(q)=1)$. For the $\mathrm{L}_{3}$-NPs, the scattering intensity, $I(q)$, was divided in two parts to model the data: one describing the inner $\mathrm{L}_{3}$ phase structure of the nanoparticles and the other one which represents the overall shape of the nanoparticles. The model described by Porcar et $a l^{30}$ (based on Lei et $a l^{31}$ and Roux et $a l .{ }^{32}$ works) was adopted to model the $\mathrm{L}_{3}$ phase inner structure. The sponge phase was here modelled by combining (a) the $\mathrm{L}_{3}$ structure factor, $S(q)_{\mathrm{L}_{3}}$, which is characterised by one or two Lorentzian broad peaks, depending on the contrast; and (b) the $\mathrm{L}_{3}$ form factor, $P(q)_{\mathrm{L}_{3}}$, described as the form factor of membrane "discoids" averaged over random orientations. Eqn (1) shows the applied $S(q)_{\mathrm{L}_{3}}$ function:

$$
S(q)_{\mathrm{L}_{3}}=\frac{A}{\frac{1}{\zeta_{1}^{2}}+\left(q-q_{1}\right)^{2}}+\frac{B}{\frac{1}{\zeta_{2}^{2}}+\left(q-q_{2}\right)^{2}}
$$

where $q_{1}$ and $q_{2}$ are the $q$ vectors of the two Bragg peaks, $A$ and $B$ Lorentzian scale factors, and $\zeta_{1}$ and $\zeta_{2}$ correlation lengths. The $P(q)_{\mathrm{L}_{3}}$ is displayed in eqn (2):

$$
P(q)_{\mathrm{L}_{3}} \approx 4\left[\pi \sigma^{2}\left(\rho_{\mathrm{b}}-\rho_{\mathrm{s}}\right)\right]^{2} \frac{\left(1-\cos \left[q t_{\mathrm{o}}\right] \exp \left[-\frac{q^{2} t_{\mathrm{o}}{ }^{2}}{32}\right]\right) / q^{2}}{q^{2} \sigma^{2}+2 \exp \left[q^{2} \sigma^{2} / 6\right]}
$$

where is $\rho_{\mathrm{b}}$ is the scattering length density (SLD) of the bilayer, $\rho_{\mathrm{s}}$ is the scattering length density of the solvent, $t_{0}$ is the membrane hydrocarbon core thickness with a Gaussian distribution of transverse SLD characterised by discoid radii of gyration of $R=\sqrt{ } 2 \cdot \sigma$, where $\sigma$ is a fitting parameter obtained from eqn (2). ${ }^{30}$ Here the scattering length density of the bilayer that makes up the sponge phase was assumed to be $1.63 \times 10^{-6} \AA^{-2}$ and $0.33 \times 10^{-6} \AA^{-2}$ for dP80 and h80 L $\mathrm{L}_{3}$-NPs, respectively. These values were estimated considering the individual amounts and SLDs of each component (Table 1). A core-shell sphere form factor, $P(q)$, was used to define the whole nanoparticle (expression is given in the ESI $\dagger$ ).

Therefore, the total scattering intensity was described as:

$$
I(q)=P(q)+S(q)_{\mathrm{L}_{3}} \cdot P(q)_{\mathrm{L}_{3}}+\text { background }
$$

Both P80 micelles and $\mathrm{L}_{3}$-NPs were considered to consist of a polydisperse core with a shell of constant thickness. This model was found to adequately describe the experimental data and the introduction of a polydisperse shell did not further improve the fit. The polydispersity of the core was described with a
Table 1 Density, volume and scattering length density (SLD) used for the analysis of SANS and neutron reflectometry data

\begin{tabular}{llcc}
\hline Compound & $\begin{array}{l}\text { Density } \\
\left(\mathrm{g} \mathrm{cm}^{-3}\right)\end{array}$ & $\begin{array}{l}\text { Volume } \\
\left(\AA^{3}\right)\end{array}$ & $\begin{array}{l}\text { SLD } \\
\left(10^{-6} \AA^{-2}\right)\end{array}$ \\
\hline DGMO & - & $735^{b}$ & 0.30 \\
GMO-50 & - & - & $0.19^{c}$ \\
55\% GMO & 0.942 & 628 & 0.21 \\
35\% GDO & 0.934 & 1104 & 0.17 \\
10\% GTO & 0.910 & 1616 & 0.15 \\
P80 & 1.064 & $2041^{d}$ & 0.55 \\
Fully protonated head & - & $1549^{e}$ & 0.79 \\
Fully deuterated head & - & 1549 & 6.90 \\
Tail & - & $492^{f}$ & -0.20 \\
dP80 (estimated) & - & - & $4.87 \pm 0.10$ \\
Lipid + hP80 & - & - & 0.33 \\
Lipid + dP80 & - & - & 1.63
\end{tabular}

${ }^{a}$ All densities were obtained from Sigma-Aldrich. ${ }^{27}{ }^{b}$ Estimated from DOPC tails divided by $2^{28}$ plus 2 glycerol groups with density of $1.26 \mathrm{~g} \mathrm{~cm}^{-3} .{ }^{c}$ Calculated from individual components indicated below. ${ }^{d}$ Estimated from known density and molecular weight. ${ }^{e}$ Volume head $=$ volume total - volume tail. ${ }^{f}$ Same DOPC tail volume ${ }^{28}$ divided by 2 , since P80 only have one hydrocarbon chain. ${ }^{g}$ Estimated from individual components and considering a DGMO/GMO-50/P80 ratio of 28/42/30 wt\%.

lognormal distribution function. For polysorbate 80 micelles, simultaneous fit of dP80 micelles in two solvent contrasts was performed. This was carried out as well for $\mathrm{L}_{3}$-NPs. We found that the fitting of the model to the data was very challenging due to the complexity of the system and the high sensitivity to the solvent contrast as the sponge phases have a very flexible structure. We therefore adopted the following approach to model the $\mathrm{L}_{3}$-NPs SANS data:

(I) Constrained and simultaneous fit of the same P80 contrast for the two dilutions studied ( 5 and 1\%) were performed in order to obtain more reliable results.

(II) In the case of the $1: 4 \mathrm{H}_{2} \mathrm{O}: \mathrm{D}_{2} \mathrm{O}$ contrast, constrained and simultaneous fit of the two equivalent samples, prepared as described in the sample preparation, was done for only $1 \%$ dilution.

The data was normalized by the volume fraction of the scattering entities prior to the fitting. All the parameters were set to be equal except scale factors, polydispersity and background. The solvent volume fraction and the scattering length density (SLD) of the lipid/P80 mixture in the core and the shell were estimated by:

(a) Using the SLD values obtained for the core and shell in different solvent contrast.

(b) Assuming that the solvent volume fraction and SLD of the lipid/P80 mixture were the same independently of the solvent. Volume fractions and SLD values for the core and the shell were considered separately.

These calculations were done comparing two isotopic solvent contrasts simultaneously, and then the results were averaged to obtain the final values. The equations used are shown in the ESI. $\dagger$ It should be noted that not all of the fits gave parameters that were physically possible. For the calculation of lipid/P80 composition and hydration it was not possible to use co-fitting for all solvent contrast used. We therefore compared calculated values for core and shell composition for a pair of two contrasts and the results are shown in Table S1 (ESI $\dagger$ ). Although most of 
values are consistent there are a few pairs of contrast that are not consistent with other values. In such a case the fits that gave the most physically reasonable parameters and gave the most consistent values of two pairs of contrasts were chosen as the best to describe the $\mathrm{L}_{3}$-NPs. Here we also benefitted from the results from the interfacial characterization as well as previous SAXS data. It is possible that a more complex model would have described the system better, but then we would have ended up with even more fitting parameters whose physical validity would be even harder to judge.

\subsection{Quartz crystal microbalance with dissipation (QCM-D)}

2.4.1. Measurements. QCM-D measurements were used to study the interfacial behaviour of $\mathrm{L}_{3}$-NPs on a $\mathrm{SiO}_{2}$ surface. The measurements were performed on a four flow cell module named E4 system (Q-Sense, Gothenburg, Sweden). QCM-D measures the change in resonance frequency $(\Delta f)$ of a quartz crystal due to the adsorption of molecules. This technique also enables to evaluate the viscoelastic properties of the adsorbed layer by measuring the change in the dissipation energy $(\Delta D)$ of the crystal. The quartz crystals used for the experiment have a fundamental frequency of $4.95 \mathrm{MHz}$ and are coated with gold and on top a $\mathrm{SiO}_{2}$ layer (QSX 303, Q-Sense) to be able to compare with the results from the neutron reflectivity study of adsorption to the spontaneously formed $\mathrm{SiO}_{2}$ layers on the silicon blocks. Before measurements, the crystals were sonicated 5 minutes with $2 \%(\mathrm{v} / \mathrm{v})$ Hellmanex III ${ }^{\circledR}$, then thoroughly rinsed with Milli-Q water and ethanol. Afterwards, they were blown dry with nitrogen and plasma cleaned (Harrick Scientific Corp, model PDC-3XG, New York, USA) for 5-10 minutes in low pressure are at 0.04 mbar just before placing them in a flow module. Then, $\mathrm{pH} 4$ or 5.5 adjusted citrate buffer solution with/without $150 \mathrm{mM} \mathrm{NaCl}$ was flowed through the cells with a peristaltic pump (Ismatec IPC-N 4, Zürich, Switzerland). The temperature of the system was set to $24{ }^{\circ} \mathrm{C}$, the fundamental frequencies and dissipation energies for each overtone were determined and the surfaces were left to equilibrate until a stable baseline was achieved. The pH-adjusted buffer with $0.1 \mathrm{mg} \mathrm{ml} \mathrm{m}^{-1}$ of $\mathrm{L}_{3}$-NPs was flowed through the measuring cells at $50 \mu \mathrm{lmin}^{-1}$ until a plateau was reached. Finally, the surfaces were rinsed with the corresponding buffer solution free of $\mathrm{L}_{3}$-NPs

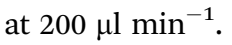

2.4.2. Data evaluation. The frequency and dissipation changes were recorded with Q-soft software and analysed with Dfind software (Q-Sense, Biolin Scientific). The analysis was performed by applying the Voigt viscoelastic model, since the linear relation between the adsorbed mass and the change in frequency is not valid for viscoelastic films. ${ }^{33}$ In these cases, there is a frequency (overtone)-dependence of the QCM response and additional energy dissipations are induced. Further details on the Voigt viscoelastic model can be found in the ESI. $\dagger$

\subsection{Neutron reflectometry (NR)}

2.5.1. Measurements. NR was performed to reveal more information about the structure formed by the $\mathrm{L}_{3}$-NPs on hydrophilic $\mathrm{SiO}_{2}$, such as layer thickness, roughness and solvent hydration. Fully hydrogenated nanoparticle dispersion was chosen for this experiment, where the sample was diluted to $1 \mathrm{mg} \mathrm{ml} \mathrm{m}^{-1}$ in deuterated $10 \mathrm{mM}$ citrate buffer at $\mathrm{pH} 4$ and $150 \mathrm{mM} \mathrm{NaCl}$. The sample was injected into the neutron reflectometry cell and left to adsorb for $30 \mathrm{~min}$, the time necessary to reach an equilibrium state as confirmed previously by QCM-D. Then, the specular reflectivity was measured before and after rinsing with deuterated buffer $\left(\mathrm{D}_{2} \mathrm{O}\right)$, and after rinsing with protonated buffer $\left(\mathrm{H}_{2} \mathrm{O}\right)$.

Polished silicon substrates, with spontaneously formed $\mathrm{SiO}_{2}$ layer (Siltronix, Archamps-France) of $8 \times 5 \times 1.5 \mathrm{~cm}$ were used and carefully cleaned before the experiment as follows: (1) at $80{ }^{\circ} \mathrm{C}$ for $5 \mathrm{~min}$ in a basic solution composed by $25 \% \mathrm{NH}_{4} \mathrm{OH}$, $30 \% \mathrm{H}_{2} \mathrm{O}_{2}$ and MilliQ water (1/1/5 volume); (2) rinsed thoroughly and several times with MilliQ water; (3) at $80{ }^{\circ} \mathrm{C}$ for $5 \mathrm{~min}$ in an acid solution composed by $32 \% \mathrm{HCl}, 30 \% \mathrm{H}_{2} \mathrm{O}_{2}$ and MilliQ water (1/1/5 volume); and (4) rinsed thoroughly with MilliQ water and kept in water until used. Just before mounting the NR cells, the silicon surfaces were rinsed with MilliQ water and $99.7 \%$ ethanol, followed by drying with $\mathrm{N}_{2}$ steam and plasma cleaning for $5 \mathrm{~min}$.

In a specular neutron reflectometry measurement, a neutron beam is reflected against an interface and the reflected neutrons are detected at the same angle as the incoming beam. The reflectivity $(R)$ is then, the intensity ratio between the incident and reflected intensity of neutrons which is measured as a function of the scattering vector $(Q)$ perpendicular to the surface:

$$
Q=\frac{4 \pi}{\lambda} \sin \theta
$$

where $\lambda$ is the neutron wavelength and $\theta$ the incident angle.

The measurements were performed at the Institute LaueLangevin (ILL, Grenoble, France) on the Super ADAM reflectometer. ${ }^{34}$ This instrument operates in a monochromatic mode at the fixed wavelength of $5.183 \AA$ and we used a $q$-range of $0 \AA^{-1}<q<0.2 \AA^{-1}$. The raw data was treated with the SuperADAM Sared program. The absolute reflectivity scale was obtained by normalizing to the direct beam at the same slit setting and then dividing by the monitor reading. This allowed us to account for fluctuations in the neutron flux and to correct for points measured for different lengths of time. Slit openings varied with the incident angle in such a way as to provide a constant illumination of the sample. Since SuperADAM is equipped with a position sensitive $2 \mathrm{D}$ detector, the background was measured simultaneously. For this purpose, the average value from the regions of interest on both sides of the specular reflected beam was used.

2.5.2. Data evaluation. The data was analysed using the Motofit software, ${ }^{35,36}$ where the reflectivity curves are fitted using an Abeles matrix method for a stratified interface. 1-Layer model was used to characterise the $\mathrm{Si}-\mathrm{SiO}_{2}$ block and a 4-layer model to define the $\mathrm{Si}-\mathrm{SiO}_{2}$ block and the lipid film formed by $\mathrm{L}_{3}$-NPs. Each layer of the model was described with a film thickness, SLD, solvent penetration (vol\%) and roughness. Different isotopic solvent contrasts were fitted simultaneously 
using the genetic algorithm in Motofit. A slightly less good fit of the data was also obtained using a 5-layer model that will be discussed in the ESI. $\dagger$

\subsection{Cryogenic transmission electron microscopy (cryo-TEM)}

The structure and morphology of the dP80 $\mathrm{L}_{3}$-NPs dispersion in water was determined by using cryo-TEM. The sample was prepared in an automatic plunge freezer (Leica EM GP) to ensure a stable temperature and to prevent the loss of water during sample preparation. The climate chamber temperature was kept at $25{ }^{\circ} \mathrm{C}$, and the relative humidity was kept close to saturation to prevent sample evaporation. The samples were prepared by placing $4 \mu \mathrm{L}$ of $5 \mathrm{wt} \% \mathrm{~L}_{3}$-NPs dispersion on glow discharged lacey formvar carbon-coated copper grids (Ted Pella and gently blotted with filter paper to obtain a thin liquid film). Immediately after being blotted, the grids were rapidly plunged into liquid ethane at $-183{ }^{\circ} \mathrm{C}$ to vitrify the water-rich samples to prevent the formation of ice crystals and to preserve the internal crystalline structure. The vitrified specimens were stored in liquid nitrogen $\left(-196{ }^{\circ} \mathrm{C}\right)$ until measurements were taken. A Fischione Model 2550 cryo transfer tomography holder was used to transfer the samples into the transmission electron microscope, a JEM-2200FS (JEOL), equipped with an in-column energy filter (Omega filter), which allows zero-loss imaging. The accelerating voltage was $200 \mathrm{kV}$ and zero-loss images were recorded with a TVIPS TemCam-F416 digital camera using Serial EM under low-dose conditions with a $10 \mathrm{eV}$ slit. The working temperature was kept below $-175{ }^{\circ} \mathrm{C}$.

\section{Results and discussion}

\subsection{Modelling lipid sponge-like nanoparticles $\left(\mathbf{L}_{3}-\mathrm{NPs}\right)$}

Small angle neutron scattering (SANS) was used to characterise the $\mathrm{L}_{3}$-NPs structure and morphology as well as to localise the polysorbate 80 (P80) on the $\mathrm{L}_{3}$-NPs. This allowed us to extract information about the role of P80 in the formation and stabilisation of these lipid sponge-like nanoparticles. Deuterated P80 (dP80) was used to highlight its location, as the scattering length density (SLD) of the deuterated head group is higher than that of the lipids and also that of hP80.

The presentation, interpretation and discussion of the data are divided in three main sections:

- The structure of the dP80 micelles: simultaneous fit of dP80 micelles in two solvent contrasts was performed using a core-shell sphere model. This allowed us also to extract the SLD of dP80.

- Revealing the inner structure of the nanoparticles: characterised by the sponge phase structure factor, $\mathrm{L}_{3}-S(q)$, and form factor, $\mathrm{L}_{3}-P(q)$.

- The overall shape and structure of the nanoparticles: described as core-shell spheres.

In order to obtain the results in Sections 3.1.2 and 3.1.3, a constrained and simultaneous fit of the model to SANS data from two samples with the same P80 contrast (either 2 dilutions or 2 sample preparations) were performed. This procedure allowed us to obtain more consistent fits as some contrast are more sensitive than others.

3.1.1. The core-shell structure of dP80 micelles: finding the degree of deuteration of dP80. The SLD and hence the degree of deuteration of P80 head groups was determined by performing SANS on the P80 micelles. Accurate determination of this value was needed to determine the location of P80 in the $\mathrm{L}_{3}$-NPs. The micelles were studied in $\mathrm{H}_{2} \mathrm{O}$ and $\mathrm{D}_{2} \mathrm{O}$ solvent contrast as depicted in Fig. 1. The data were fitted to a core-shell sphere model (Table 2). The core comprises the hydrocarbon tail while the shell is composed by the head group. Amani et al. ${ }^{37}$ performed molecular dynamics simulation of polysorbate 80 in a micelle in water and they found acyl chain lengths of about $22 \AA$, which fits well with average core radius of $23 \AA$ we observed (Table 2). The corresponding length of the polyethylene group was found to be around $15 \AA$, which is less than the shell thickness of about $22 \AA$ we observed. This discrepancy can possibly be assigned to the inherent polydispersity of P80. Based on the molecular structure of P80 one would expect that the complete hydrogenated and deuterated P80 head groups have a SLD of $0.793 \times 10^{-6} \AA^{-2}$ and $6.903 \times 10^{-6} \AA^{-2}$, respectively. From the fitted parameters we estimated that the SLD of dP80 head group was $6.48 \times 10^{-6} \AA^{-2}$, which corresponds to a degree of deuteration of $93 \pm 2 \%$. Therefore, the SLD of the entire dP80 molecule was estimated to be $4.87 \times 10^{-6} \AA^{-2}$. Further details

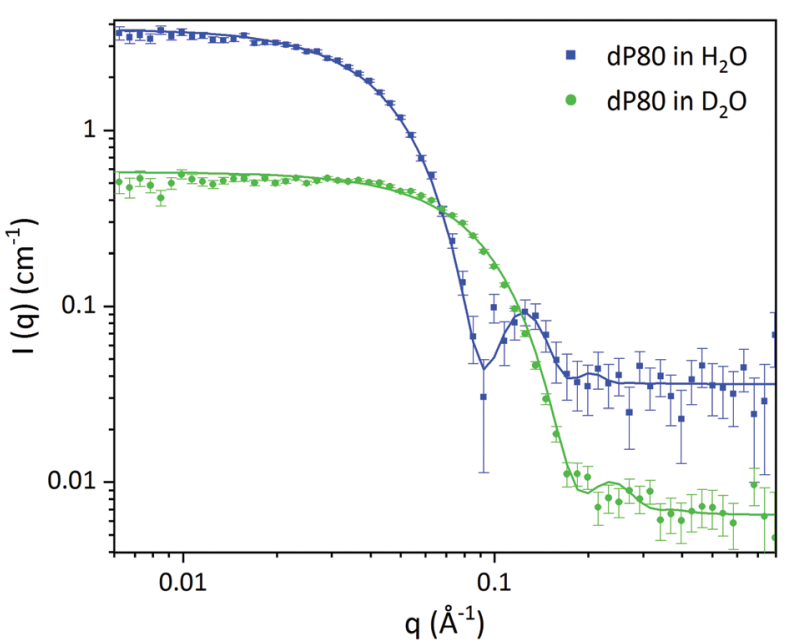

Fig. 1 SANS data of $d P 80$ micelles in $\mathrm{H}_{2} \mathrm{O}$ (blue) and $\mathrm{D}_{2} \mathrm{O}$ (green). Solid lines represent the core-shell sphere fitted model from Table 2.

Table 2 Core-shell sphere models for dP80 micelles depicted in Fig. 1

\begin{tabular}{lll}
\hline $\mathrm{dP} 80$ micelles & $\mathrm{D}_{2} \mathrm{O}$ & $\mathrm{H}_{2} \mathrm{O}$ \\
\hline
\end{tabular}

Volume fraction of micelles (\%) $\quad 4.00 \pm 0.03$ Average core diameter $(\AA) \quad 46.0 \pm 0.3$

Core polydispersity $\quad 0.100 \pm 0.004$

Shell thickness $(\AA)$

SLD core $\left(\AA^{-2}\right) 10^{-6}$

SLD shell $\left(\AA^{-2}\right) 10^{-6}$

SLD solvent $\left(\AA^{-2}\right) 10^{-6}$

Solvent in core (vol\%)

Solvent in the shell (vol\%)

Background $\left(\mathrm{cm}^{-1}\right)$
$21.6 \pm 0.2$

$2.00 \pm 0.01$

$6.38 \pm 0.01$

6.35

$0.10 \pm 0.02$

$27.5 \pm 0.3$

$75.8 \pm 0.3$

0.0065
$1.14 \pm 0.02$

$-0.56$

0.036 
on the fitted and theoretical values used can be found in Tables 1 and 2 .

3.1.2. The lipid sponge $\left(L_{3}\right)$ phase: inner particle structure. As discussed in the experimental section, the scattering from the inner and overall structure of the particles contribute to the recorded SANS data. In this section we focus on the $q$-range of $q \geq 0.02 \AA^{-1}$. This part of the scattering curve is particularly influenced by the $\mathrm{L}_{3}$ phase, which is dominant at this particle composition as previously shown from our SAXS and cryo-TEM results. ${ }^{13}$ Fig. 2 depicts the experimental and fitted scattering curves of $\mathrm{L}_{3}$-NPs with hP80 and dP80 at different solvent

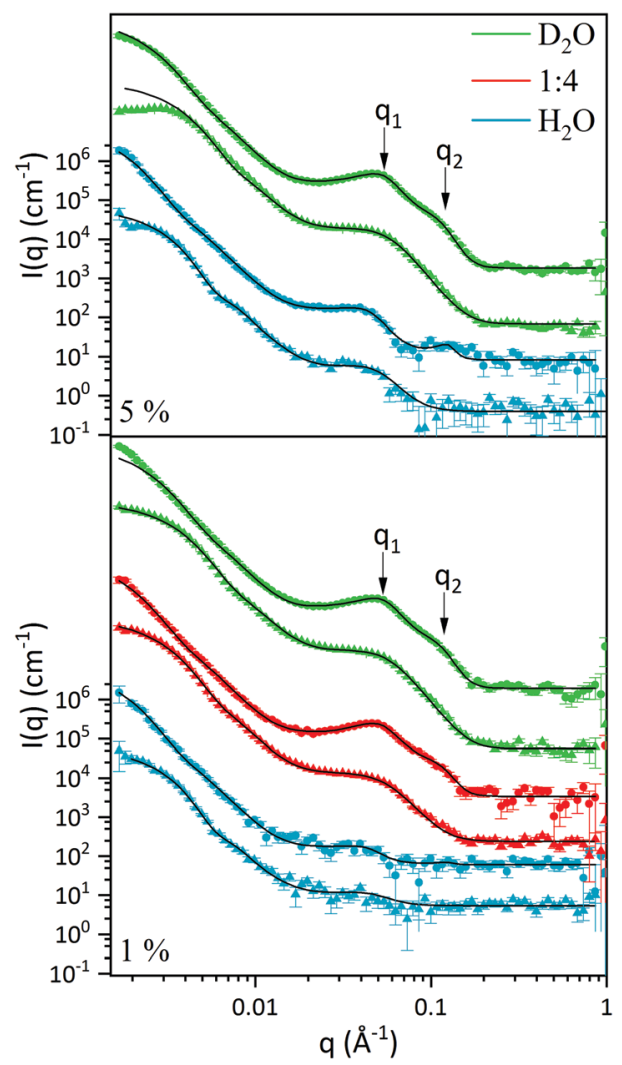

Fig. 2 SANS curves of $L_{3}$-NPs with their corresponding fitted curve (solid line), as a function of P80 and solvent contrast. hP80 and dP80 $\mathrm{L}_{3}$-NPs are depicted as triangles and circles, respectively, while $\mathrm{H}_{2} \mathrm{O}, 1: 4 \mathrm{H}_{2} \mathrm{O}: \mathrm{D}_{2} \mathrm{O}$ and $\mathrm{D}_{2} \mathrm{O}$ solvent contrast are in blue, red and green, respectively. Top plot shows non-diluted samples $(5 \%)$, while bottom are diluted to $1 \%$. The $y$-axis scale, indicated for the lowest scattering curve, are the same for all the curves. For the sake of clarity the other scattering curves are shifted upwards without rescaling. contrast and dilutions. A visual inspection of the data shows clear differences between the results from the two P80 contrast. Two broad peaks can be distinguished for the sample with dP80, while samples with hP80 only exhibit the lower $q$ broad peak, around $0.05 \AA^{-1}$. The $q$ position of the first broad peak $\left(q_{1}\right)$ reflects the $\mathrm{L}_{3}$ cell-cell correlation length while the second one $\left(q_{2}\right)$ is related to lipid bilayer distances. ${ }^{14,31,32}$ This clearly indicates that P80 is involved in the formation of bicontinuous sponge bilayers. However, the P80 location in the bilayer can only be seen when emphasized by deuteration of the molecule, i.e. when the contrast between the head group and the acyl chain is large. Further discussion on this matter will be brought up later when considering the overall particle arrangement.

In order to find a realistic model to fit the data recorded in this $q$-range, we used a combination of sponge phase structure and form factors $\left(S(q)_{\mathrm{L}_{3}}\right.$ and $P(q)_{\mathrm{L}_{3}}$, respectively) as described in the experimental section and previous work. ${ }^{30}$

3.1.2.1. $L_{3}$ correlation peak, $S(q) L_{3}$. Table 3 shows the results obtained from the fitting, where the peaks' maxima were fixed. The $q_{1}$ value was found to be $0.05 \AA^{-1}$, which agrees quite well with the SAXS results where $q_{1}$ was $0.047 \AA^{-1}$. It should be noted that, in spite of the fact that the dP80 and hP80 used here were from a different batch than the hP80 used for the SAXS and cryo-TEM experiments, ${ }^{13}$ we still found similar sponge phases in terms of dimensions and location in the phase diagram. Comparing different solvent contrast, it can be seen that in $\mathrm{H}_{2} \mathrm{O}$ the repeat distances are larger than in $\mathrm{D}_{2} \mathrm{O}$, as $q_{1}$ shift towards lower $q$-values, both for dP80 and hP80. The effect is larger for dP80 than for hP80. This could possibly be due to the sample in $\mathrm{H}_{2} \mathrm{O}$ being more hydrated than the ones with $\mathrm{D}_{2} \mathrm{O}$. However, it should be noted that the shift of $q_{2}$ is in the opposite direction.

3.1.2.2. Discoids form factor, $P(q)_{L_{3}}$. The bilayer form factor in the bicontinuous $\mathrm{L}_{3}$ phase was described using the one of discoids averaged over random orientations, as used previously by Porcar et al. ${ }^{30}$ The hydrocarbon core thickness was found to be around 24.5 and $18.8 \AA$ for dP80 and hP80 samples, respectively. Since the dP80 tail extension was found to be $23 \AA$ in the micelles and the hydrocarbon region is the same for DGMO and GMO-50, these results suggest that the lipid and P80 tails are somehow interdigitated or tilted in the $\mathrm{L}_{3}$ bilayer. The lower value for the hP80 sample might be due to the smaller difference in SLD between head group and tail.

Table 3 Eqn (1) and (2) fits of the inner sponge phase structure (shown in Fig. 2) for $\mathrm{L}_{3}$-NPs with deuterated and protonated P80 (dP80 and hP80, respectively) in $\mathrm{D}_{2} \mathrm{O}, 1: 4 \mathrm{H}_{2} \mathrm{O}: \mathrm{D}_{2} \mathrm{O}$ and $\mathrm{H}_{2} \mathrm{O}$

\begin{tabular}{|c|c|c|c|c|c|c|}
\hline Solvent contrast & \multicolumn{3}{|c|}{ dP80 L $\mathrm{L}_{3}$-NPs } & \multicolumn{3}{|c|}{ hP80 L $\mathrm{L}_{3}$-NPs } \\
\hline$t_{0}(\AA)$ & $24.8 \pm 0.4$ & $26.0 \pm 0.3$ & $22.6 \pm 2.8$ & $18.0 \pm 0.1$ & $18.1 \pm 0.6$ & $20 \pm 3$ \\
\hline$q_{1}\left(\AA^{-1}\right)$ & 0.0524 & 0.05 & 0.0432 & 0.05 & 0.0497 & 0.045 \\
\hline$\zeta_{1}(\AA)$ & $61.4 \pm 0.5$ & $58.3 \pm 1.1$ & $67.7 \pm 4.2$ & $33.1 \pm 0.6$ & $30.7 \pm 0.6$ & $44 \pm 13$ \\
\hline$q_{2}\left(\AA^{-1}\right)$ & 0.109 & 0.115 & 0.126 & & & \\
\hline$\zeta_{2}(\AA)$ & $26.9 \pm 0.6$ & $27 \pm 2$ & $59 \pm 19$ & & & \\
\hline
\end{tabular}




\subsubsection{The overall shape of the nanoparticles}

3.1.3.1. Differences and similarities between hP80 and $d P 80$ $L_{3}$-NPs. A core-shell sphere model was used to fit the SANS data for the low $q$-range, i.e. $q<0.02 \AA^{-1}$ (Fig. 2). The model was chosen taking into account the sponge-like nanoparticle morphology previously observed by cryo-TEM, ${ }^{13}$ where the core is characterised by a denser $\mathrm{L}_{3}$ phase while the surface presented swollen bilayer structures. Inter-particle interactions were assumed to be negligible as the concentration of particle was considered to be sufficiently low. Discrepancies could, however, be seen when comparing fitted curves at very low $q$-values of diluted and non-diluted samples.

Tables 4 and 5 show the results from the fitting, as well as estimated solvent hydration and SLD-solvent corrected values for the core and the shell of $\mathrm{L}_{3}$-NPs. The first aspect that can be noticed is the difference in size between the samples that contain hydrogenated and deuterated P80. In fact, $\mathrm{L}_{3}$-NPs with hP80 are smaller and present lower polydispersity. This sizedependence can also be appreciated from Fig. 2 where samples with hP80 reach a plateau at low $q$-values that is not present in dP80 samples. These discrepancies could be related to the degree of polymerization of P80 which makes the dP80 and hP80 chemically different. Here it should be noted that the P80 synthesis is very challenging and often give non-negligible differences between batches and there is also the issue of chemical stability. These aspects are discussed in detail by Kishore et $a .^{38}$ As a result, dP80 samples seem to be more prone to aggregation than hP80 nanoparticles, which could also explain why they are more polydisperse (as also shown in Fig. 3). Therefore, nanoparticles with dP80 and hP80 were considered separately. However, we note that when it comes to phase behaviour, the different P80 samples investigated do not give any large variation.

As a general trend, the core dimension of $\mathrm{L}_{3}$-NPs increases with the $\mathrm{H}_{2} \mathrm{O}$ content while the shell thickness was largest for the $1: 4 \mathrm{H}_{2} \mathrm{O}: \mathrm{D}_{2} \mathrm{O}$ solvent contrast and the decreases with $\mathrm{D}_{2} \mathrm{O}$ followed by $\mathrm{H}_{2} \mathrm{O}$. This could be related to the dilutions used for each simultaneous fitting. While for $\mathrm{D}_{2} \mathrm{O}$ and $\mathrm{H}_{2} \mathrm{O}$ contrast two dilutions where used ( $5 \%$ and $1 \%$ ), only one dilution $(1 \%)$ was used for $1: 4 \mathrm{H}_{2} \mathrm{O}: \mathrm{D}_{2} \mathrm{O}$ solvent contrast. The results suggest that the dilution process has an effect on the shell thickness, while the core diameter is less affected by it. This also shows that the inner sponge phase structure can only uptake a certain amount of solvent. Despite this size difference likely associated with dilution, we were able to obtain good fits for $5 \%$ and $1 \% \mathrm{~L}_{3}$-NPs in $\mathrm{D}_{2} \mathrm{O}$ and $\mathrm{H}_{2} \mathrm{O}$.

The results from fitting the core shell model to the experimental data shows that the solvent contrast has a huge impact on the SLD of the core and the shell. This is expected as the sponge phase is an open and flexible system composed of a curved lipid bilayer with interconnected solvent channels that are connected to the bulk solvent. Therefore, when the bulk solvent's contrast changes, the contrast of the solvent inside the sponge mesh changes accordingly. It was found that the solvent present in the core was 59 and $56 \%$ for hP80 and dP80 $\mathrm{L}_{3}$-NPs, respectively. This agrees quite well with previous findings that showed that at this DGMO/GMO-50/P80 composition the lipid system could uptake around $60 \%$ of water. ${ }^{13}$ In addition, the solvent content in the shell was found to be around $90 \%$, which means that the contrast between the shell and the surrounding medium is low. This can be explained by the swollen structures observed in the $\mathrm{L}_{3}$-NPs surface (Fig. 3 and Valldeperas et $a .^{13}{ }^{13}$ ) These structures appear to be composed of curved layers that enhance the contact with the bulk solvent as the surface area to volume ratio is increased. Therefore, it is expected that they are highly hydrated. Interestingly, the $\mathrm{L}_{3}$-NPs in water that contain dP80 show a less swollen surface structure and a denser core than the one we reported previously for hP80 $\mathrm{L}_{3}$-NPs. ${ }^{13}$ These highly swollen surfaces in the hP80 nanoparticles implies a large amount of solvent in that area, which makes it harder to see in SANS due to the lack of contrast between shell and medium.

Table 4 Core-shell sphere fits used to describe the overall shape of the $\mathrm{L}_{3}-\mathrm{NPs}$ with deuterated and protonated P80 (dP80 and hP80, respectively) in $\mathrm{D}_{2} \mathrm{O}, 1: 4 \mathrm{H}_{2} \mathrm{O}: \mathrm{D}_{2} \mathrm{O}$ and $\mathrm{H}_{2} \mathrm{O}$ reported in Fig. 2

\begin{tabular}{|c|c|c|c|c|c|c|}
\hline \multirow[b]{2}{*}{ Solvent contrast } & \multicolumn{3}{|l|}{ dP80 L $\mathrm{L}_{3}$-NPs } & \multicolumn{3}{|l|}{$\underline{\mathrm{hP} 80 \mathrm{~L}_{3} \text {-NPs }}$} \\
\hline & $\mathrm{D}_{2} \mathrm{O}$ & $1: 4 \mathrm{H}_{2} \mathrm{O}: \mathrm{D}_{2} \mathrm{O}$ & $\mathrm{H}_{2} \mathrm{O}$ & $\mathrm{D}_{2} \mathrm{O}$ & $1: 4 \mathrm{H}_{2} \mathrm{O}: \mathrm{D}_{2} \mathrm{O}$ & $\mathrm{H}_{2} \mathrm{O}$ \\
\hline Average core diameter $(\AA)$ & $706 \pm 2$ & $950 \pm 2$ & $967 \pm 12$ & $492 \pm 1$ & $586 \pm 1$ & $662 \pm 8$ \\
\hline Core polydispersity & 0.3 & 0.3 & 0.3 & 0.3 & $0.22 / 0.28$ & 0.19 \\
\hline Shell thickness $(\AA)$ & $173 \pm 4$ & $248 \pm 6$ & $126 \pm 19$ & $82 \pm 2$ & $116 \pm 1$ & $50 \pm 8$ \\
\hline SLD core $\left(\AA^{-2}\right) 10^{-6}$ & $4.00 \pm 0.03$ & $3.22 \pm 0.05$ & $0.15 \pm 0.02$ & $3.85 \pm 0.05$ & $3.03 \pm 0.02$ & $-0.197 \pm 0.003$ \\
\hline SLD shell $\left(\AA^{-2}\right) 10^{-6}$ & $6.07 \pm 0.01$ & $4.83 \pm 0.06$ & $-0.10 \pm 0.04$ & $5.74 \pm 0.04$ & $4.510 \pm 0.003$ & $-0.44 \pm 0.06$ \\
\hline SLD solvent $\left(\AA^{-2}\right) 10^{-6}$ & 6.35 & 4.968 & -0.56 & 6.35 & 4.968 & -0.56 \\
\hline Background $\left(\mathrm{cm}^{-1}\right)$ & 0.897 & 1.11 & 0.667 & 0.695 & 1.78 & 0.35 \\
\hline
\end{tabular}

Table 5 Estimated values of solvent volume fraction and scattering length density (SLD) corrected by the solvent contribution on the core and the shell of $d P 80$ and $h P 80 L_{3}-N P s$

\begin{tabular}{|c|c|c|}
\hline & hP80 L $\mathrm{L}_{3}$-NPs & dP80 $\mathrm{L}_{3}$-NPs \\
\hline Average solvent hydration in the core (vol\%) & $58.8 \pm 0.6$ & $55.8 \pm 0.4$ \\
\hline Average solvent hydration in the shell (vol\%) & $89.50 \pm 0.01$ & $90.6 \pm 0.3$ \\
\hline Average SLD core $\left(\AA^{-2}\right) 10^{-6}-$ solvent corrected & $0.27 \pm 0.08$ & $1.01 \pm 0.04$ \\
\hline Average SLD shell $\left(\AA^{-2}\right) 10^{-6}-$ solvent corrected & $0.57 \pm 0.01$ & $3.45 \pm 0.07$ \\
\hline
\end{tabular}




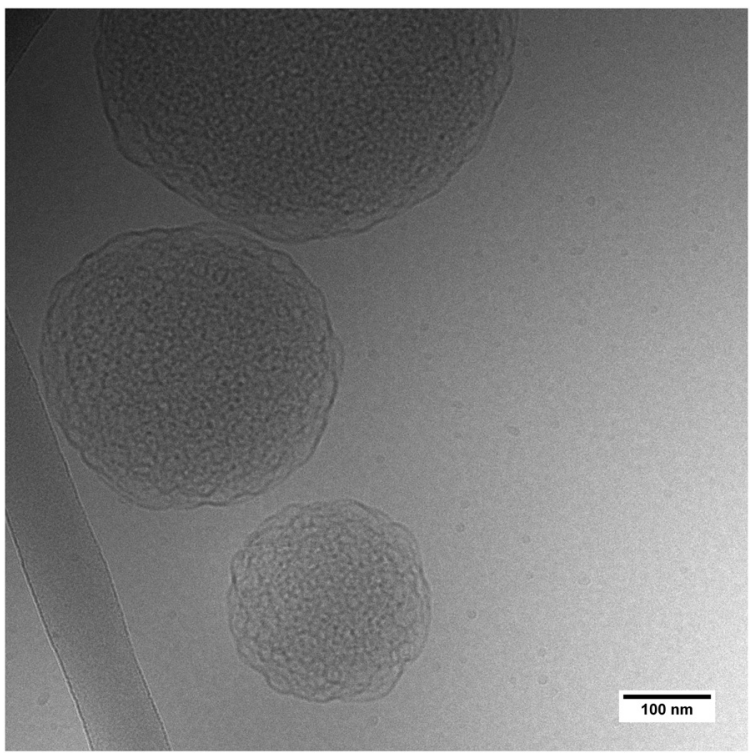

Fig. 3 Cryo-TEM image of $\mathrm{L}_{3}-\mathrm{NPs}$ with dP8O in $\mathrm{H}_{2} \mathrm{O}$ solvent. Note that the sample shown here is from a different batch than the SANS sample.

3.1.3.2. $d P 80$ stabilizer mostly found on the $L_{3}$-NPs surface. We previously noted that dP80 was not affecting the sponge correlation peak, but we also saw that the second $q_{2}$ peak was only visible for particles with dP80. This suggested that P80 somehow participates in the formation of the structure.

The data for hP80 $\mathrm{L}_{3}$-NPs does not allow us to draw any firm conclusions regarding the location of P80 due to the lack of contrast between lipids and hP80. However, it is interesting to notice that the SLD values for the lipid/surfactant mixture in the core and the shell are rather close, i.e. 0.27 and $0.57 \times 10^{-6} \AA^{-2}$, for the non-deuterated nanoparticles (Table 5). While the core SLD is slightly below the expected value for a homogeneous particle $\left(0.33 \times 10^{-6} \AA^{-2}\right)$, the shell SLD is very close to the hP80 SLD value of $0.55 \times 10^{-6} \AA^{-2}$ (Table 1 ). This suggests that $L_{3}$-NPs shell is mostly formed by hP80 at the expense of an enriched lipid core. However, the contrast is not large enough to confirm that there is less than $30 \mathrm{wt} \%$ of P80 in the core.

In the case of $\mathrm{dP} 80 \mathrm{~L}_{3}$-NPs, it was observed that the SLD of the lipid/surfactant components in the core $\left(1.01 \times 10^{-6} \AA^{-2}\right)$ is much lower compared to that of the shell $\left(3.45 \times 10^{-6} \AA^{-2}\right)$. Since the lipids are not deuterated, these high SLD values can only be attributed to dP80. This suggests that deuterated P80 is mostly found on the $\mathrm{L}_{3}$-NPs surface, i.e. stabilizing the lipid nanoparticles and allowing the formation of more swollen bilayers. Moreover, the core SLD value indicates that there is also some dP80 that contributes to the core sponge phase structure, as the SLD is higher than in the non-deuterated system. It is worth noting that the core SLD is lower than the expected SLD of the lipid + P80 mixture $\left(1.63 \times 10^{-6} \AA^{-2}\right)$, indicating that not all the P80 is in the core and thus, supporting the observations made for the hP80 nanoparticles. Therefore, it can be confirmed that the sponge-like nanoparticles present phase separation with a rich P80 shell. This is in line with what Wadsäter et $a l^{22}$ previously reported for cubic micellar type of liquid crystalline nanoparticles. Their study focused on the effect of P80 concentration on soy phosphatidylcholine (SPC)/ glycerol dioletate (GDO) lipid liquid crystalline nanoparticles. They observed that by increasing the dP80, the shell thickness was larger and richer in P80 at the expense of a GDO enriched core. In addition, the internal structure of the nanoparticles was affected at larger amounts (10 and $15 \mathrm{wt} \%$ ) of P80, indicating that it was not only on the surface but also on the core of the particles. Yepuri et al. compared the cubic phase nanoparticle structures of phytantriol with two non-ionic stabilizers Tween ${ }^{\circledR}$ 80 (or P80) and Pluronic ${ }^{\circledR}$ F127. ${ }^{39}$ The location of the dispersing agent was revealed by SANS using deuterated phytantriol. They found that for P80, the surfactant molecules in the phytantriol-d/ Tween ${ }^{\circledR} 80$ followed a self-avoiding walk organization, whereas F127 in phytantriol-d/Pluronic ${ }^{\circledR}$ F127 particles appeared in a more rod-like packing. Here we note that in our case the more even distribution of P80 is favored by the fact that the lipids used and P80 has the same acyl chain.

\subsection{Adsorption of $L_{3}$-NPs on hydrophilic silica}

Adsorption experiments were carried out to determine the interfacial properties of $\mathrm{L}_{3}$-NPs with hydrogenated P80. The aim was to understand their colloidal stability and how they interact with a silica interface. This will allow us to understand the driving force for the interaction as well as what happens with $\mathrm{L}_{3}$-NPs when they attach to a surface. Apart from the fundamental aspects, this is directly relevant when these types of dispersions are kept in glass vials for storage purposes. Here we used a negatively charged $\mathrm{SiO}_{2}$ (hydrophilic) as model substrate.

3.2.1. Quartz crystal microbalance with dissipation. QCM-D was used to study the adsorption kinetics at different conditions, as well as to evaluate the viscoelastic properties of the lipid interfacial film. Previously it has been demonstrated that QCM-D is also a powerful tool to reveal the fate of soft nanoparticles, like vesicles, when they attach to a surface. ${ }^{40}$ In addition, the interfacial wet mass, i.e. the adsorbed mass including the solvent coupled to the film, could be estimated. This was obtained by fitting the data using the Voigt viscoelastic model as described above.

The first experiment carried out was to study the adsorption of $\mathrm{L}_{3}$-NPs from pure water with no addition of any salt $(\mathrm{pH} \approx$ 6.5-7), as they are prepared and stored under these conditions. Fig. S3a (ESI $\dagger$ ) shows that no adsorption was observed which could be the result of electrostatic repulsion between the negatively charged $\mathrm{L}_{3}$-NPs and silica. Similar results were reported by Vandoolaeghe et $a l .{ }^{19}$ who studied the affinity of GMO/ Pluronic F127 cubosomes to hydrophilic $\mathrm{SiO}_{2}$ surfaces in water and at different $\mathrm{pH}$ values and ionic strengths. They found that by reducing the $\mathrm{pH}$ or adding electrolytes, adsorption of cubosomes took place. Therefore, we followed the same approach by studying the adsorption under low $\mathrm{pH}$ conditions $(\mathrm{pH}=4$ and 5.5) with and without $150 \mathrm{mM} \mathrm{NaCl}$.

The results shown in Fig. 4 and Fig. S2b-d (ESI $\dagger$ ), confirm that under these conditions $\mathrm{L}_{3}$-NPs adsorb, although no major differences in frequency and dissipation changes were observed when comparing the different solution conditions. We here focus on the results for $\mathrm{pH}=4$ with $150 \mathrm{mM}$, where we expect 


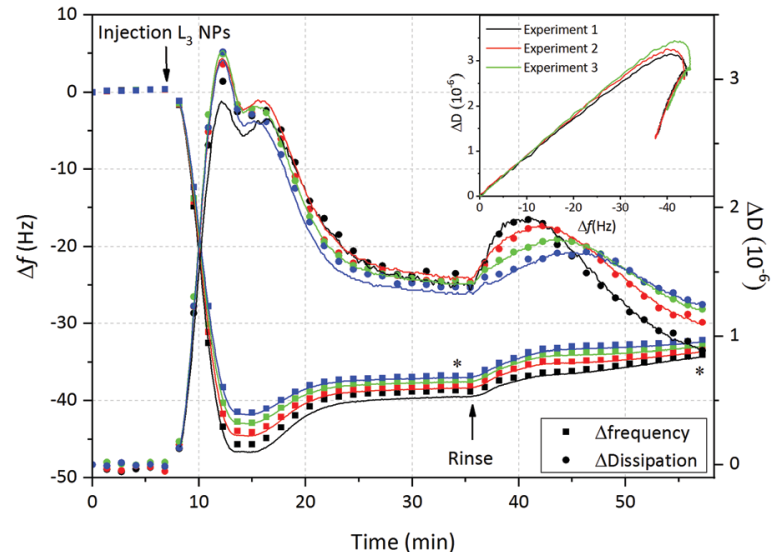

Fig. 4 Change in frequency ( $\Delta f$, squares) and dissipation ( $\Delta D$, circles) as a function of time after addition of $0.1 \mathrm{mg} \mathrm{ml}^{-1} \mathrm{~L}_{3}$-NPs on hydrophilic silica. Measurements were performed at $10 \mathrm{mM}$ citrate buffer at $\mathrm{pH}=4$ and $150 \mathrm{mM} \mathrm{NaCl}$. Experimental (dots) and fitted data (solid line) are depicted for four different overtones (5, black; 7, red; 9, green; and 11, blue). Embedded plot shows the $\Delta D$ vs. $\Delta f$ for three equivalent experiments.

that the adsorption should not be hampered by repulsion between particles and the surface.

Fig. 4 displays the change in frequency $(\Delta f)$ and dissipation $(\Delta D)$ as a function of time after addition of sponge-like nanoparticles on hydrophilic $\mathrm{SiO}_{2}$. After injection there is an immediate decrease of $\Delta f$ followed by a minimum and then a plateau at slightly higher frequency, which is reached after $30 \mathrm{~min}$. This is accompanied by initially high $\Delta D$ values which are reduced once the steady state is achieved. Taking into account that the $\mathrm{L}_{3}$-NPs were initially $180 \mathrm{~nm}$, we could suggest that, as soon as they are in contact with the hydrophilic $\mathrm{SiO}_{2}$, the lipid nanoparticles spread (at least partially) and reorganize themselves forming a lipid + P80 film characterised by the decrease in dissipation and mass (increase in $\Delta f$ ). This is supported by the kink observed in the $\Delta D v s$. $\Delta f$ plot (embedded in Fig. 4). Similar observations and conclusion regarding change in the soft particle structure on the surface has been previously reported for vesicles. ${ }^{40}$ It is not surprising that the sponge-like nanoparticles show similarities to the vesicles, as the sponge phase is formed by curved bilayers and it is close to the lamellar phase as showed in the bulk phase behaviour. ${ }^{13}$ Based on the experimental observation we suggest that the adsorption process is divided in two steps: (1) rapid adsorption of $\mathrm{L}_{3}$-NPs material and (2) rearrangement of material previously adsorbed. Furthermore, it was possible to rule out the adsorption of intact nanoparticles. However, it is difficult to confirm it with QCM-D and neutron reflectometry experiments were therefore conducted to give more insight into this matter.

Once the steady state was reached, the surface was rinsed with the same buffer at a higher flow rate $\left(200 \mu \mathrm{l} \mathrm{min}{ }^{-1}\right)$. It could be observed on Fig. 4 that the frequency slowly increases, while the dissipation presents an initial rise that is reduced to values slightly below the plateau value. This dissipation bump was not observed when the rinsing flow rate was slower, although dissipation was somewhat reduced (data not shown). These results could suggest that: (a) the hydration of the layer decreases and a more rigid layer is formed and/or (b) excess of $\mathrm{L}_{3}$-NPs, lipid or P80 not attached directly to the surface are washed off. What is clear is that part of the lipid + P80 layer remains on the surface and is hardly disrupted when rinsing, especially when the rinsing flow rate is lower.

Finally, the Voigt model was used to determine the interfacial wet mass (and thickness) after $\mathrm{L}_{3}$-NPs adsorption, as the dissipation values were slightly too high to apply the Sauerbrey equation, indicating that the film formed on hydrophilic silica presented viscoelastic properties. Four overtones were used in order to obtain a good fit of the data that includes the adsorption and rinsing steps. Table 6 shows the thickness and wet mass values at the steady state and after rinsing (values averaged from three repeat experiments). These values also reflect the loss in mass after rinsing, with final values of $655 \mathrm{ng} \mathrm{cm}-2$ and 67 A for adsorbed mass and thickness, respectively. QCM-D does not give information about the morphology of the film and thus, neutron reflectometry is needed. However, we can speculate that the lipid + P80 film on hydrophilic $\mathrm{SiO}_{2}$ could form a bilayer, as we previously found that for bulk inverse bicontinuous cubic phases $\left(\approx 50 \mathrm{wt} \% \mathrm{H}_{2} \mathrm{O}\right)$ the bilayer thickness was $46 \AA^{13}{ }^{13}$ SAXS results shows that the $\mathrm{L}_{3}$-NPs have a more swollen bicontinuous structure. ${ }^{13}$ Therefore, they can be expected to give a larger thickness value of surface layer formed from the $\mathrm{L}_{3}$-NPs, which is consistent with QCM-D data (Table 6). Furthermore, QCM-D thickness/ adsorbed mass data also include the water coupled to the film.

3.2.2. Neutron reflectometry. Neutron reflectometry provides information about the lipid film structure, including thickness, roughness, composition and solvent penetration into the lipid layer. First, the $\mathrm{Si}_{-} \mathrm{SiO}_{2}$ block was characterised at two different isotopic solvent contrasts $\left(\mathrm{D}_{2} \mathrm{O}\right.$ and $\left.\mathrm{H}_{2} \mathrm{O}\right)$. Then, $1 \mathrm{mg} \mathrm{ml}^{-1}$ of $\mathrm{L}_{3}$-NPs in deuterated buffer was introduced to the neutron reflectivity cell and left to adsorb for at least $30 \mathrm{~min}$. After this time, a measurement was carried out before rinsing with $\mathrm{D}_{2} \mathrm{O}$ buffer and finally, $\mathrm{H}_{2} \mathrm{O}$ buffer. The measuring time was about $2-3 \mathrm{~h}$ depending on the contrast. Additional scans were performed to verify that the plateau value was reached. In addition, the curve after rinse was identical to the one recorded after adsorption.

As NR is less sensitive than QCM-D and the NR surface area was larger, a higher concentration of $\mathrm{L}_{3}$-NPs was used in order to ensure full coverage of the surface. $\mathrm{A}_{3}$-NPs concentration of $1 \mathrm{mg} \mathrm{ml}{ }^{-1}$ was enough to observe a fringe in NR, as the sample was left to adsorb for $30 \mathrm{~min}$, while in QCM-D the sample was constantly flowed passed the substrate.

Fig. 5 shows the NR data after adsorption of $\mathrm{L}_{3}$-NPs. The data clearly show a strong adsorption of particles as a fringe appears, which is not present for bare silica in $\mathrm{D}_{2} \mathrm{O}$ buffer. The corresponding reflectivity curves in the $\mathrm{H}_{2} \mathrm{O}$ solvent contrast is also significantly

Table 6 Thickness and wet mass values obtained using the Voigt viscoelastic model after addition of $\mathrm{L}_{3}$-NPs on hydrophilic silica

\begin{tabular}{lll}
\hline Period & $\begin{array}{l}\text { Thickness } \\
(\AA)\end{array}$ & $\begin{array}{l}\text { Wet mass } \\
\left.(\mathrm{ng} \mathrm{cm})^{-2}\right)\end{array}$ \\
\hline After injection of $\mathrm{L}_{3}$-NPs (plateau) & $80 \pm 4$ & $789 \pm 42$ \\
After rinsing with buffer & $67 \pm 3$ & $655 \pm 30$
\end{tabular}




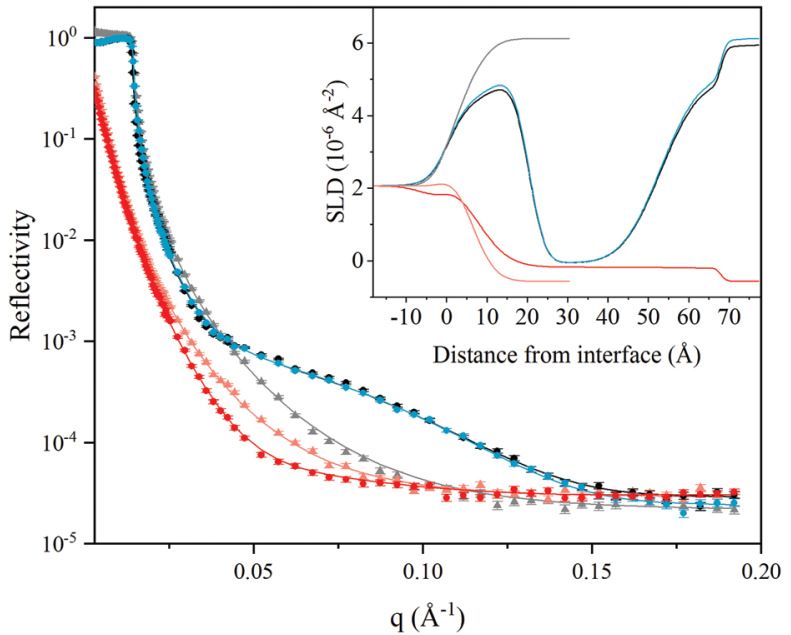

Fig. 5 Neutron reflectivity curves as a function of momentum transfer (q) before addition of $\mathrm{L}_{3}-\mathrm{NPs}$ (grey for $\mathrm{D}_{2} \mathrm{O}$ and light red for $\mathrm{H}_{2} \mathrm{O}$ ), after adsorption of $\mathrm{L}_{3}$-NPs in deuterated buffer (black) and after rinsing with deuterated (blue) and protonated water (red). Experimental and fitted data are depicted as dots and solid line respectively. Embedded plot show the scattering length density (SLD) values as a function of distance from the interface obtained from the fitting.

different. Interestingly, the curves before and after rinsing in $\mathrm{D}_{2} \mathrm{O}$ contrast do not show much difference, which suggests that the interfacial lipid film structure is not altered by rinsing and that $1 \mathrm{mg} \mathrm{ml} \mathrm{m}^{-1}$ of $\mathrm{L}_{3}$-NPs was enough to obtain full coverage. This supports what was proposed in the analysis of the QCM-D data: that excess material is not in direct contact with the surface and could be easily removed without affecting the interfacial layer. However, we could not exclude the adsorption of intact nanoparticles onto the lipid film. The minor differences observed were mostly attributed to a change in the SLD of the solvent, as the injected $\mathrm{L}_{3}$-NPs contain some $\mathrm{H}_{2} \mathrm{O}$ inside the structure that is eliminated by rinsing.

Simultaneous fitting of a four-layer model to the data was performed. The results are presented as the SLD profile inserted in Fig. 5 and the fitting parameters are summarised in Table 7. The four-layer model consist of: (1) $\mathrm{SiO}_{2}$; (2) lipid head groups; (3) lipid hydrocarbon tails and; (4) lipid head groups, where the inner and outer head groups were considered equivalent in terms of thickness and SLD. The total thickness of the lipid layers formed from the $\mathrm{L}_{3}$-NPs dispersion was found to be $63 \AA$ bilayer structure on hydrophilic silica, which agrees quite well with QCM-D values after rinsing. Table 7 shows the results obtained from the fit. It is noteworthy that the head group is highly hydrated $(\approx 80 \%)$ and it is smaller than the dP80 head group observed by SANS (23 $\AA$ ). Taking into account that the

Table 7 Parameters used to fit the neutron reflectometry data displayed on Fig. 5

\begin{tabular}{lcccc}
\hline & $\begin{array}{l}\text { Thickness } \\
(\AA)\end{array}$ & $\begin{array}{l}\text { SLD } \\
\left(10^{-6} \AA^{-2}\right)\end{array}$ & $\begin{array}{l}\text { Solvent } \\
(\text { vol\%) }\end{array}$ & \multicolumn{1}{l}{$\begin{array}{l}\text { Roughness } \\
(\AA)\end{array}$} \\
\hline $\mathrm{SiO}_{2}$ & $5 \pm 1.0$ & \multicolumn{1}{c}{3.41} & $7 \pm 2$ & $3 \pm 2$ \\
Lipid head & $15 \pm 1$ & $1.24 \pm 0.1$ & $80 \pm 5$ & $7.5 \pm 0.2$ \\
Lipid tail & $33 \pm 3$ & $-0.16 \pm 0.1$ & $1.78 \pm 4$ & $3.1 \pm 5$ \\
Lipid head & $15 \pm 1$ & $1.24 \pm 0.1$ & $80 \pm 5$ & $7.5 \pm 0.2$
\end{tabular}

head group of DGMO and GMO-50 are smaller and that the thickness values are averaged over the whole surface, it makes sense that the observed thickness is reduced. In the case of the hydrocarbon tails, there is almost no solvent, as expected because of its hydrophobicity, and present a thickness of $33 \AA$. This value is slightly larger than the ones found for the $\mathrm{L}_{3}$-NPs studied by SANS. This suggests that the interfacial bilayer is less compact than the bilayers in $\mathrm{L}_{3}$-NPs. The tail layer thickness is lower than twice the dP80 tail size and thus supports the fact that the tails might be interdigitated or tilted. SLD values for lipid heads and tails agree quite well with the values expected (see Table 1 for more detailed information on head and tail SLD values of each component).

The surface excess $(\Gamma)$ of each layer was estimated in order to compare the neutron reflectivity with the QCM-D values. To do so, the following equation was used:

$$
\Gamma=\frac{d \cdot \Phi \cdot M}{V_{\mathrm{M}} N_{\mathrm{A}}}
$$

where $d$ is the thickness, $\Phi$ is the volume fraction of the lipid + P80, $M$ is the molar mass, $V_{\mathrm{M}}$ the molecular volume and $N_{\mathrm{A}}$ the Avogadro number. $d$ and $\Phi$ were taken from the NR fit, while $M$ and $V_{\mathrm{M}}$ were estimated considering a DGMO/GMO-50/P80 weight ratio of $28 / 42 / 30$ and values from Table 1 . The total adsorbed mass obtained was $325.3 \mathrm{ng} \mathrm{cm}{ }^{-2}$, where $34.6 \mathrm{ng} \mathrm{cm} \mathrm{cm}^{-2}$ was for each head group layer and $256.1 \mathrm{ng} \mathrm{\textrm {cm } ^ { - 2 }}$ for the hydrocarbon tail. In contrast, the QCM-D values after rinsing were $655 \mathrm{ng} \mathrm{cm}{ }^{-2}$, which is almost double of the estimated value from NR. This was expected as the mass measured by the QCM-D technique includes the water within the lipid film, while that from NR does not. This is supported as well by the highly hydrated head group layers obtained from the neutron reflectometry fits.

With the experiments performed here it is not possible to distinguish the location of P80 on the interfacial film, as dP80 was not used for these experiments. However, we could speculate that P80 might play an important role in the adsorption process of $\mathrm{L}_{3}$-NPs. Earlier it was suggested ${ }^{24,41,42}$ that attractive interactions exist between the polyethylene oxide segments of the P80 head group and $\mathrm{SiO}_{2}$. Since P80 is mostly found on the spongelike nanoparticles surface, the adsorption driving force could be the same here, as no significant changes were obtained between different conditions studied by QCM-D.

\section{Conclusion}

In this work the role of P80 in the formation of sponge-like nanoparticles was investigated by SANS. It was found that the $\mathrm{L}_{3}$-NPs features local phase separation, as the more swollen bilayer type of structure on the particle surface is rich in P80 at the expense of a more DGMO/GMO-50 rich core. The sponge phase inner structure was however retained and was found to be very similar to the one reported earlier. ${ }^{13}$ This confirms our hypothesis that the polymer polysorbate 80 not only contributes to the formation of highly swollen inverse bicontinuous phases, but also acts as a stabilizer when these LLC phases are in excess water. Therefore, polysorbate 80 plays an important role here and confers long term stability to these nanoparticles. 
The interfacial behaviour of $\mathrm{L}_{3}$-NPs was studied by QCM-D and neutron reflectometry. The results reveal that particles spread to form a relatively hydrated $65 \AA$ thick film on hydrophilic silica. Thus, the layer thickness is much lower than the particle size confirming the flexibility of the particles.

These results are of great interest since highly swollen sponge phase nanoparticles can be used as delivery systems, e.g. of bioactive macromolecules. The adsorption data suggests that the particles collapse once they are adsorbed and that is likely to lead to release of the cargo. We envisage that by tuning the stabiliser surface interactions we can potentially achieve targeted release.

\section{Conflicts of interest}

There are no conflicts to declare.

\section{Acknowledgements}

This work was financially supported by the BIBAFOODs project from the European Union's Seventh Framework Programme FP7/2007-2013. We thank the ISIS Neutron and Muon source (UK) and the Super ADAM project at Institut Laue-Langevin (ILL, France), supported by Swedish Research council, for allocating beamtime at SANS2D and SuperADAM beamline, respectively. The deuterated and hydrogenated Tween 80 samples were kindly provided by Dr Robert K. Thomas, University of Oxford, UK. The authors are grateful for the SASView support from Paul Butler and valued discussion provided by Paul Butler and Marc Obiols-Rabasa. This work benefited from the use of the SasView application, originally developed under NSF award DMR-0520547.

\section{References}

1 K. Larsson, J. Phys. Chem., 1989, 93, 7304-7314.

2 K. Larsson, Curr. Opin. Colloid Interface Sci., 2000, 5, 64-69.

3 K. Larsson, P. Quinn, K. Sato and F. Tiberg, Lipids. Structure, physical properties and functionality, The Oily Press, Bridgwater, England, 2006.

4 J. Gustafsson, H. Ljusberg-Wahren, M. Almgren and K. Larsson, Langmuir, 1996, 12, 4611-4613.

5 J. Barauskas, A. Misiunas, T. Gunnarsson, F. Tiberg and M. Johnsson, Langmuir, 2006, 22, 6328-6334.

6 A. Angelova, B. Angelov, R. Mutafchieva, S. Lesieur and P. Couvreur, Acc. Chem. Res., 2011, 44, 147-156.

7 L. Sagalowicz and M. E. Leser, Curr. Opin. Colloid Interface Sci., 2010, 15, 61-72.

8 M. Malmsten, J. Dispersion Sci. Technol., 2007, 28, 63-72.

9 C. J. Drummond and C. Fong, Curr. Opin. Colloid Interface Sci., 1999, 4, 449-456.

10 M. Caffrey, Curr. Opin. Struct. Biol., 2000, 10, 486-497.

11 C. E. Conn and C. J. Drummond, Soft Matter, 2013, 9, 3449.

$12 \mathrm{~J}$. Barauskas and T. Nylander, Delivery and Controlled Release of Bioactives in Foods and Nutraceuticals, Woodhead Publishing Limited, Abington, England, 2008, pp. 107-131.
13 M. Valldeperas, M. Wiśniewska, M. Ram-On, E. Kesselman, D. Danino, T. Nylander and J. Barauskas, Langmuir, 2016, 32, 8650-8659.

14 B. Angelov, A. Angelova, R. Mutafchieva, S. Lesieur, U. Vainio, V. M. Garamus, G. V. Jensen and J. S. Pedersen, Phys. Chem. Chem. Phys., 2011, 13, 3073-3081.

15 H. M. G. Barriga, A. I. I. Tyler, N. L. C. McCarthy, E. S. Parsons, O. Ces, R. V. Law, J. M. Seddon and N. J. Brooks, Soft Matter, 2015, 11, 600-607.

16 A. I. I. Tyler, H. M. G. Barriga, E. S. Parsons, N. L. C. McCarthy, O. Ces, R. V. Law, J. M. Seddon and N. J. Brooks, Soft Matter, 2015, 11, 3279-3286.

17 P. Vandoolaeghe, A. R. Rennie, R. A. Campbell and T. Nylander, Langmuir, 2009, 25, 4009-4020.

18 D. P. Chang, J. Barauskas, A. P. Dabkowska, M. Wadsäter, F. Tiberg and T. Nylander, Adv. Colloid Interface Sci., 2015, 222, 135-147.

19 P. Vandoolaeghe, F. Tiberg and T. Nylander, Langmuir, 2006, 22, 9169-9174.

20 A. J. Tilley, C. J. Drummond and B. J. Boyd, J. Colloid Interface Sci., 2013, 392, 288-296.

21 Y.-D. Dong, I. Larson, T. J. Barnes, C. A. Prestidge, S. Allen, X. Chen, C. J. Roberts and B. J. Boyd, Langmuir, 2012, 28, 13485-13495.

22 M. Wadsäter, J. Barauskas, S. Rogers, M. W. A. Skoda, R. K. Thomas, F. Tiberg and T. Nylander, Soft Matter, 2015, 11, 1140-1150.

23 P. Vandoolaeghe, R. A. Campbell, A. R. Rennie and T. Nylander, J. Phys. Chem. C, 2009, 113, 4483-4494.

24 D. P. Chang, M. Jankunec, J. Barauskas, F. Tiberg and T. Nylander, ACS Appl. Mater. Interfaces, 2012, 4, 2643-2651.

25 M. Younus, A. Hawley, B. J. Boyd and S. B. Rizwan, Colloids Surf., B, 2018, 169, 135-142.

26 I. M. Tucker, J. T. Petkov, J. Penfold, R. K. Thomas, P. Li, A. R. Cox, N. Hedges and J. R. P. Webster, J. Phys. Chem. B, 2014, 118, 4867-4875.

27 Sigma-Aldrich, https://www.sigmaaldrich.com, accessed July 2018.

28 J. F. Nagle and S. Tristram-Nagle, Biochim. Biophys. Acta, Rev. Biomembr., 2000, 1469, 159-195.

29 SasView, https://www.sasview.org/, accessed July 2018.

30 L. Porcar, W. A. Hamilton, P. D. Butler and G. G. Warr, Langmuir, 2003, 19, 10779-10794.

31 N. Lei, C. R. Safinya, D. Roux and K. S. Liang, Phys. Rev. E: Stat. Phys., Plasmas, Fluids, Relat. Interdiscip. Top., 1997, 56, 608-613.

32 D. Roux, M. E. Cates, U. Olsson, R. C. Ball, F. Nallet and A. M. Bellocq, Europhys. Lett., 1990, 11, 229-234.

33 F. Höök, B. Kasemo, T. Nylander, C. Fant, K. Sott and H. Elwing, Anal. Chem., 2001, 73, 5796-5804.

34 A. Vorobiev, A. Devishvilli, G. Palsson, H. Rundlöf, N. Johansson, A. Olsson, A. Dennison, M. Wollf, B. Giroud, O. Aguettaz and B. Hjörvarsson, Neutron News, 2015, 26, 25-26.

35 A. Nelson, J. Phys.: Conf. Ser., 2010, 251, 012094.

36 A. Nelson, J. Appl. Crystallogr., 2006, 39, 273-276.

37 A. Amani, P. York, H. de Waard and J. Anwar, Soft Matter, 2011, 7, 2900-2908. 
38 R. S. K. Kishore, S. Kiese, S. Fischer, A. Pappenberger, U. Grauschopf and H.-C. Mahler, Pharm. Res., 2011, 28, 1194-1210.

39 N. R. Yepuri, A. J. Clulow, R. N. Prentice, E. P. Gilbert, A. Hawley, S. B. Rizwan, B. J. Boyd and T. A. Darwish, J. Colloid Interface Sci., 2019, 534, 399-407.
40 E. Reimhult, F. Höök and B. Kasemo, J. Chem. Phys., 2002, 117, 7401-7404.

41 O. Svensson, K. Thuresson and T. Arnebrant, Langmuir, 2008, 24, 2573-2579.

42 D. P. Chang, M. Jankunec, J. Barauskas, F. Tiberg and T. Nylander, Langmuir, 2012, 28, 10688-10696. 